\title{
A Lagrangian Stochastic Model for the concentration fluctuations
}

\author{
L. Mortarini ${ }^{1,3}$ and E. Ferrero ${ }^{2,3}$ \\ ${ }^{1}$ Dipartimento di Fisica Generale, Università di Torino, v. P. Giuria 1, 10125, Torino, Italy \\ ${ }^{2}$ Dipartimento di Scienze e Tecnologie Avanzate, Univ. del Piemonte Orientale, Via Bellini, 25/G 15100 Alessandria, Italy \\ ${ }^{3}$ ISAC-CNR, Corso Fiume 4, 10133, Torino, Italy
}

Received: 21 July 2004 - Published in Atmos. Chem. Phys. Discuss.: 2 June 2005

Revised: 13 September 2005 - Accepted: 13 September 2005 - Published: 23 September 2005

\begin{abstract}
A Lagrangian Stochastic Model for the twoparticles dispersion, aiming at simulating the pollutant concentration fluctuations, is presented. Three model versions (1-D, 2-D and 3-D) are tested. Firstly the ability of the model to reproduce the two-particle statistics in a homogeneous isotropic turbulence is discussed, comparing the model results with theoretical predictions in terms of the probability density function (PDF) of the particles separation and its statistics. Then, the mean concentration and its fluctuations are considered and the results presented. The influence of the PDF of the particle separation on the concentration fluctuations is shown and discussed. We found that the separation PDF in the inertial subrange is not gaussian and this fact influences the predicted concentration fluctuations.
\end{abstract}

\section{Introduction}

Lagrangian Stochastic Models (LSM) are based on the Langevin equation, which allows describing the temporal evolution of the velocity of pollutant particles in a turbulent field. The solution of the Langevin equation is a continuous stochastic Markov process. In fact, particle position and velocity, in a turbulent flow, can be considered a bivariate Markov process in the range of the spectrum between the Kolmogorov time scale and the velocity correlation Lagrangian time scale $\mathrm{T}_{\mathrm{L}}$.

Thomson (1987) provides a complete theory of the LSM based on the concept of the Markovian process. In that paper he introduced the so called "well mixed condition" as a basic constraint in order to assess the validity and physical consistency of a dispersion model. He also demonstrated that this condition is satisfied by his model.

Correspondence to: L. Mortarini

(1.mortarini@ isac.cnr.it)
Many numerical models have been developed based on this fundamental work aiming to simulate the dispersion of pollutants in the atmospheric boundary layer in different stability conditions (see for instance Luhar and Britter, 1989; Hurley and Physick, 1991; Tinarelli et al., 1994; Du et al., 1994). These models are able to account for higher order turbulence moments of the atmospheric PDF and complex turbulence flow dynamics (Ferrero et al., 2003), and have demonstrated to be able to accurately reproduce the mean concentration field of the dispersed tracer.

It should be stressed that the one-particle model is only able to describe the absolute dispersion and to predict the mean concentration fields. When one is interested in the relative dispersion and fluctuation concentration field, a twoparticle model should be developed and applied.

Many authors attempt to extend the results obtained with the single particle dispersion models to those for the particle pairs suggesting heuristic models (Durbin, 1980; Sawford, 1984; Kaplan and Dinar, 1989). However, more recently, Thomson (1990) and Borgas and Sawford (1994) following a more rigorous approach, based on the stochastic processes theory, prescribed a complete three dimensional model for two-particles dispersion in homogeneous isotropic turbulence.

An important advantage of the two-particle model is the ability to include second order chemical reactions (Crone et al., 1999; van Dop, 2001). Unfortunately a unique solution of the Fokker-Planck equation does not exist for the twoparticle model, even in one dimension and in isotropic turbulence (Sawford, 1993).

A two-particles LSM for relative dispersion in homogeneous and isotropic turbulence is presented. In particular, the behavior of the separation and barycentre of the particles positions generated by the model are analysed and compared with the Taylor (1921) theory for the Lagrangian dispersion and its extension to the relative dispersion (Lee and Stone, 1983; Borgas and Sawford, 1994). The form of the

(C) 2005 Author(s). This work is licensed under a Creative Commons License. 
separation PDF's is also investigated and compared with the classical theory (Richardson, 1926). Finally the mean concentration and the concentration fluctuations predicted by the 1-D, 2-D and 3-D versions of the model are compared with those predicted by analytical formulas assuming different PDFs and experimental data.

\section{The model}

In order to obtain a model for particle pairs, the Eulerian PDF should depend on the particle relative separation $(\Delta)$. The simplest choice is to assume $P_{E}(\Delta, u)$ to be Gaussian, depending on a six-dimensional velocity covariance tensor. The PDF is completely determined if all the components are known. These components are determined, in homogeneous isotropic turbulence, following Thomson (1990) and depend on the correlation functions (Durbin, 1980).

The general equations of the model are:

$$
\begin{array}{r}
d u_{i}(t)=a_{i}(u, t) d t+\delta_{i j} \sqrt{C_{0} \epsilon} d W_{j} \\
d x_{i}(t)=u_{i}(t) d t
\end{array}
$$

where $i=1, . ., 6$ corresponds to the three coordinates for each of the particles of a couple, $C_{0}$ is the Kolmogorov constant, $\epsilon$ the dissipation rate of the turbulent kinetic energy, $d W_{j}$ is a Gaussian white noise and

$a_{i}(u, t)=\frac{\sigma^{2}}{T_{L}} V_{i j}^{-1} u_{j}+\frac{1}{2} V_{l j}^{-1} \frac{\partial V_{i l}}{\partial x_{k}} u_{j} u_{k}$

where $\sigma$ is the turbulent velocity field standard deviation, $T_{L}$ is the Lagrangian time scale and $V$ is

$V=\left\langle\boldsymbol{u}_{i} \boldsymbol{u}_{j}\right\rangle=\left(\begin{array}{c}\left\langle\boldsymbol{u}_{i^{\prime}}^{(1)} \boldsymbol{u}_{j^{\prime}}^{(1)}\right\rangle\left\langle\boldsymbol{u}_{i^{\prime}}^{(1)} \boldsymbol{u}_{j^{\prime \prime}}^{(2)}\right\rangle \\ \left\langle\boldsymbol{u}_{i^{\prime \prime}}^{(2)} \boldsymbol{u}_{j^{\prime}}^{(1)}\right\rangle\left\langle\boldsymbol{u}_{i^{\prime \prime}}^{(2)} \boldsymbol{u}_{j^{\prime \prime}}^{(2)}\right\rangle\end{array}\right)$

where 1,2 indicate the particle $\left(i^{\prime}, j^{\prime}, i^{\prime \prime}, j^{\prime \prime}=1,2,3\right)$.

Following Thomson (1990), we introduce the new coordinates: separation and the barycentre,

$$
\left\{\begin{array}{l}
\Delta=\frac{x^{(1)}-x^{(2)}}{\sqrt{2}} \\
\Sigma=\frac{x^{(1)}+x^{(2)}}{\sqrt{2}}
\end{array}\right.
$$

Equation (1) can be replaced by the Langevin equations for the separation and barycentre velocities, which can be found by solving the corresponding Fokker-Planck equation. With the aim of assessing the possibility to use simplified versions of the complete 3-D model in simulating relative dispersion we derived the 1-D and 2-D solution. In the 1-D case (Ferrero and Mortarini, 2005) we have:

$$
\begin{aligned}
& d u_{\Delta}=-\frac{1}{2}\left(\frac{C_{0} \epsilon}{\sigma^{2}}+\frac{d f}{d \Delta} u_{\Delta}\right) \frac{u_{\Delta} d t}{(1-f(\Delta))}+\sqrt{C_{0} \epsilon} d W \\
& d u_{\Sigma}=-\frac{1}{2}\left(\frac{C_{0} \epsilon}{\sigma^{2}}-\frac{d f}{d \Delta} u_{\Delta}\right) \frac{u_{\Sigma} d t}{(1+f(\Delta))}+\sqrt{C_{0} \epsilon} d W
\end{aligned}
$$

where $f(\Delta)$ is the longitudinal velocity correlation function which is prescribed as in Thomson (1990).

It can be noted that only the equation for $u_{\Delta}$ (6) accounts for the particle-particle interaction $\left(u_{\Delta} u_{\Delta}=\frac{1}{2}\left(u_{1}^{2}-2 u_{1} u_{2}+u_{2}^{2}\right)\right)$. On the contrary the non linear term in the $u_{\Sigma}$ Eq. (7) does not depend on this effect $\left(u_{\Sigma} u_{\Delta}=\frac{1}{2}\left(u_{1}^{2}-u_{2}^{2}\right)\right)$. As a matter of fact, Thomson (1990) has pointed out that the model does not satisfy the "reduction to one particle" condition: fixing the initial position of the first particle of a pair, the ensemble of the trajectories of this particle does not depend on the initial position of the second particle.

It should be also stressed that, in order to overcome the lack in simulating the molecular diffusion which acts at the very small scale, the model requires a finite initial distance between the particles.

\section{Relative dispersion}

In order to test the model, firstly we compared its results with the theoretical predictions prescribed by Borgas and Sawford (1991). They proposed the following trend for the particles separation mean square in a moving set of coordinates independent from the initial velocity:

$$
<\Delta^{2}>= \begin{cases}\frac{1}{3} C_{0} \epsilon t^{3} & t_{\eta} \ll t \ll t_{0} \\ \frac{1}{3}\left(C_{0}-\gamma\right) \epsilon t^{3} & t_{0} \ll t \ll T_{L}\end{cases}
$$

where $t_{\eta}$ is the Kolmogorov length scale and $t_{0}$ depends on the initial separation $\Delta_{0}$ :

$t_{0}=\left(\Delta_{0}^{2} / \epsilon\right)^{\frac{1}{3}}$.

It represents the time at which the motion of the particles becomes independent from the initial separation (Batchelor, 1952).

The presence of the additional term $\gamma$ is due to the crosscorrelation terms in the correlation tensor (4) (Borgas and Sawford, 1991), whose effect appears only for lager separation.

Figure 1 shows the separation standard deviation as a function of time predicted by the 3-D model compared with the theoretical prediction (8). It demonstrates the presence of an intermediate sub-range inside the inertial range, whose amplitude depends on the initial separation of the particles. For time greater than $t_{0}$ the separation mean square grows with a different trend according to Eq. (8).

This behavior can be interpreted as follows. Immediately after the release $\left(t \ll t_{0}\right)$ the separation of the particles induced by the turbulent eddies is less than the initial separation $\left(\Delta_{0}\right)$ of the particles of the pair. Only when the separation becomes greater $\left(t>t_{0}\right)$ the effect of the particle correlation can be observed. For great initial separation the particles are already uncorrelated at the beginning of the simulation and hence follow the single particle dynamics. 


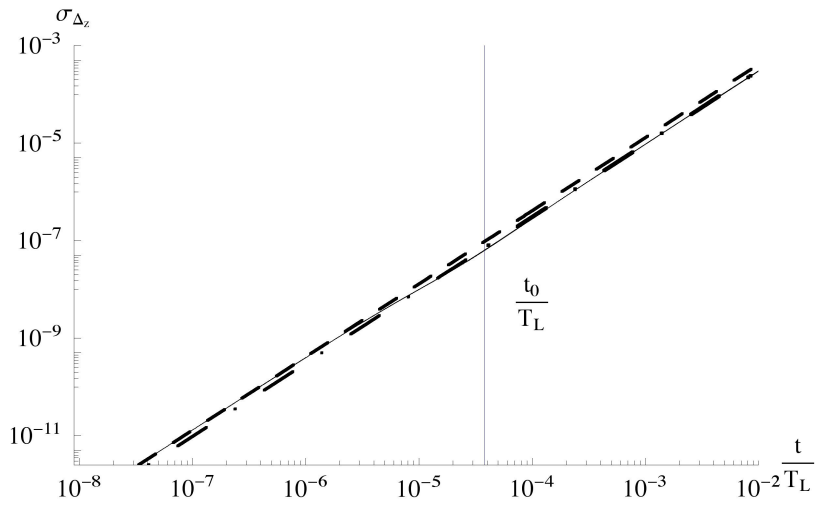

Fig. 1. Separation's standard deviation vs normalised time. Solid line: model; dashed line: first of Eq. (8) square root; dash-dotted line: second of Eq. (8).

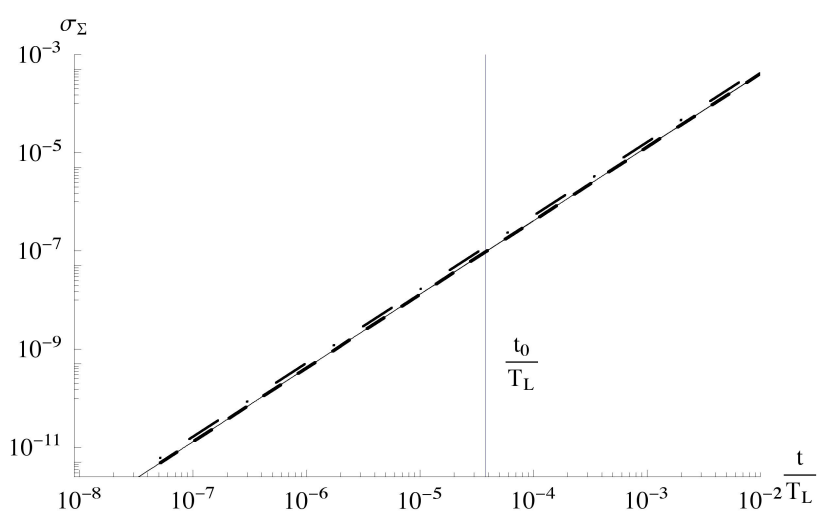

Fig. 2. Barycentre's standard deviation vs normalised time. Solid line: model; dashed line: first of Eq. (10) square root; dash-dotted line: second of Eq. (10).

A similar formula can be derived for the barycentre's mean square:

$<\Sigma^{2}>= \begin{cases}\frac{1}{3} C_{0} \epsilon t^{3} & t_{\eta} \ll t \ll t_{0} \\ \frac{1}{3}\left(C_{0}+\gamma\right) \epsilon t^{3} & t_{0} \ll t \ll T_{L}\end{cases}$

The comparison between theoretical prediction (10) and the 3-D model results is shown in Fig. 2.

It can be seen that the barycentre is insensitive to the particle-particle interaction and it follows the same trend in all the inertial range.

As we stated in a previous article (Ferrero and Mortarini, 2005), the different behaviour between the separation and the barycentre standard deviations (Eqs. 8 and 10) is due to the non linear terms in the Langevin Eqs. (6) and (7) respectively. As a matter of fact, the term $u_{\Delta} u_{\Delta}$ in (6) contains the interaction of the particles $u_{1} u_{2}$, while the term $u_{\Delta} u_{\Sigma}$ in (7) lacks this part. When the intial separation memory is lost, the correlation between the particles influences only the separation and not the barycentre. This unbalanced behaviour is due
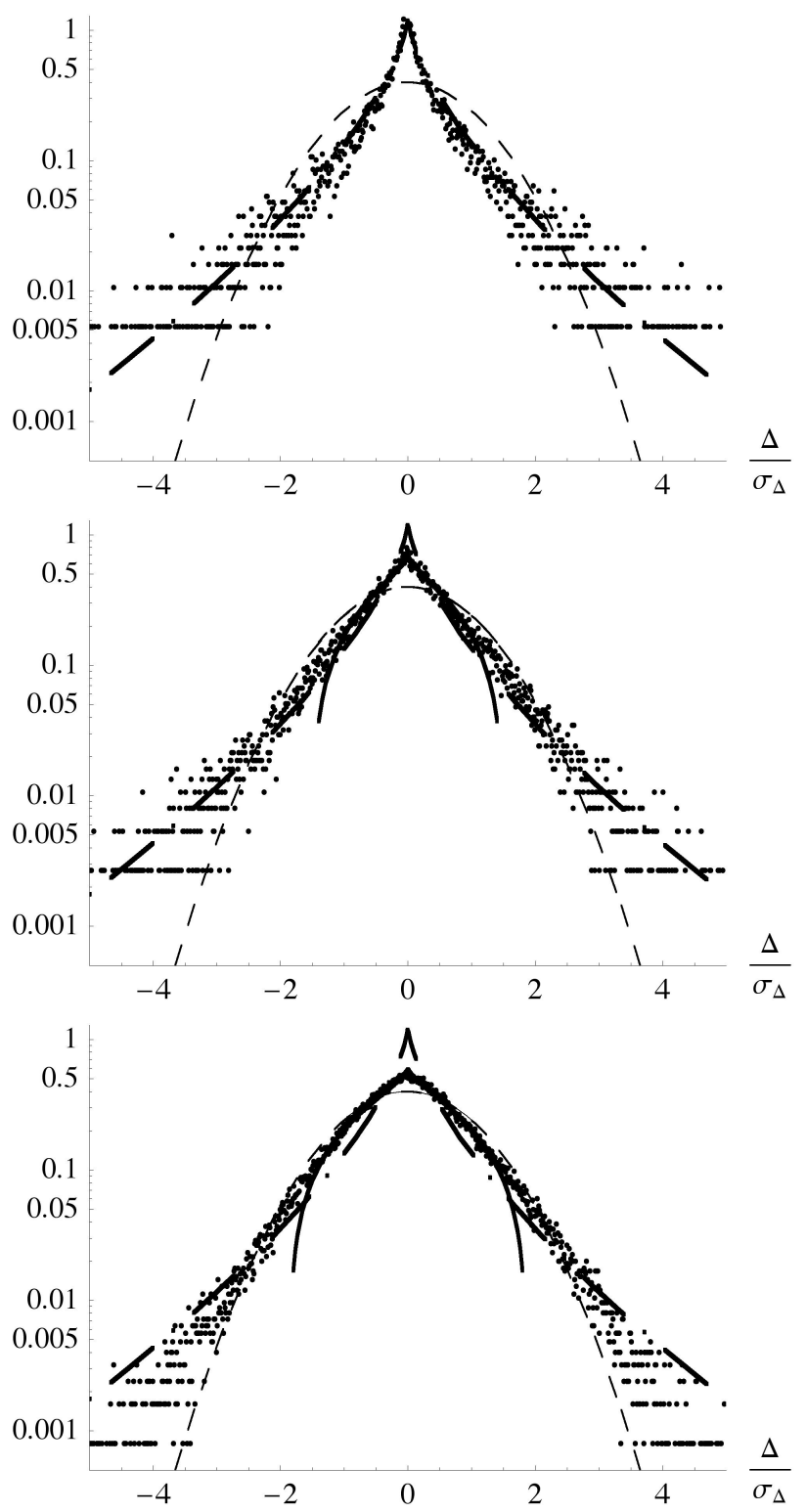

Fig. 3. $P(\Delta, s \mid t)$ produced by the models (dots) in the intermediate range in the (from top) 1-D, 2-D and 3-D cases. Dashed line: Gaussian; dash-dotted line: Richardson; solid line: Eq. (13).

to the model failing of the reduction to one particle criteria (Thomson, 1990), if the barycentre standard deviation slope doesn't balance the separation's one, the single particle standard deviation can not follow the theoretical prediction as long as:

$\left\langle X^{2}\right\rangle=\frac{1}{2}\left(\left\langle\Delta^{2}\right\rangle+\left\langle\Sigma^{2}\right\rangle\right)$.

While the single particle position and barycentre PDF's are generally accepted to be Gaussian, there is no definitive theory on the shape of the separation PDF within the inertial subrange. Richardson (1926) proposed a form for the pair 


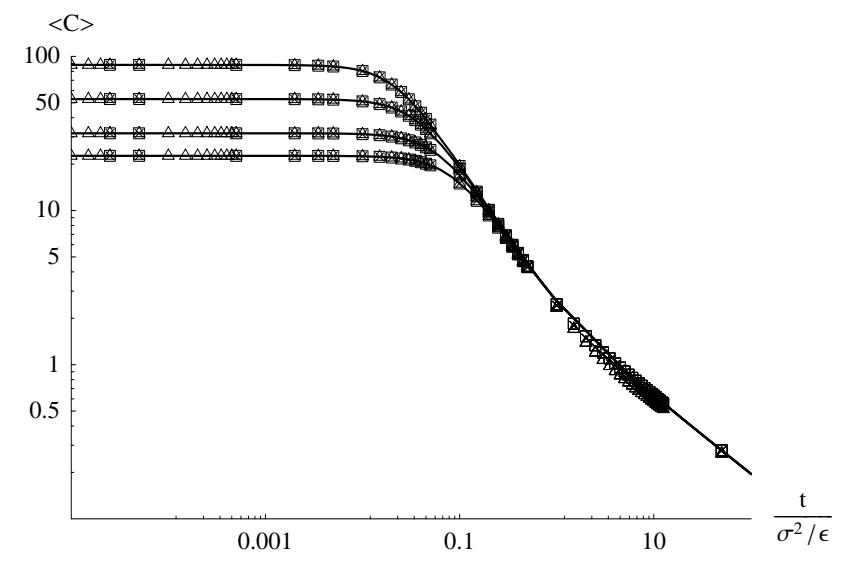

Fig. 4. Comparison between mean concentrations predicted by the 1-D $(\triangle), 2-\mathrm{D}(\times)$ and 3-D $(\square)$ models and theoretical prediction (contonous lines) for different source sizes, where the intial mean concentration is inversely proportional to the source size.

separation PDF, whose one-dimensional expression is:

$P(\Delta \mid x, t)=\frac{9}{4 \sqrt{\pi}}(\eta t)^{-3 / 2} e^{-\frac{9 \Delta^{2 / 3}}{4 \eta t}}$

where $\eta$ depends on the Richardson constant $g$ and on the rate of dissipation of turbulent kinetic energy $\epsilon$ through the relation: $\eta=\left(\frac{243}{560} g \epsilon\right)^{1 / 3}$.

A different form of the PDF was suggested by Thomson (1990) following (Monin and Yaglom, 1975, p. 384) characterized by a less sharp maximum and small tails:

$P(\Delta \mid x, t) \simeq \alpha-\beta \Delta^{2 / 3}$

where $\alpha$ is the variance of the hypothetical concentration field and $\beta$ is proportional to its rate of dissipation (Thomson, 1990).

It can be easily noticed that Thomson PDF (Eq. 13) is the first order expansion of the Richardson PDF (Eq. 12) and that is not a proper PDF as long as does not tend to zero for $\Delta \rightarrow \infty$, but can be considered as a theoretical trend of the particle PDF separation when the particles are very close. This approximated expression should fit the PDF only for small separations in the inertial subrange.

Looking at the modelled PDF for the 1-D, 2-D and 3-D cases, we see that, in the first case it fits the Richardson prediction, while, increasing the dimensions of the space where the particles move and hence their degrees of freedom, the PDF becomes less sharp and show a good agreement only with the first order expansion (Eq. 13) of the Richardson PDF, which departs from the Gaussian statistic as well.

The separation PDFs in the intermediate range $t_{0} \ll t \ll T_{L}$ for the 1-D, 2-D and 3-D cases are shown in Fig. 3. The PDF produced by the 1-D model has a more sharp maximum and agrees better with the Richardson PDF. In the 2-D and 3-D cases the PDFs show a smoother maxima but still they are

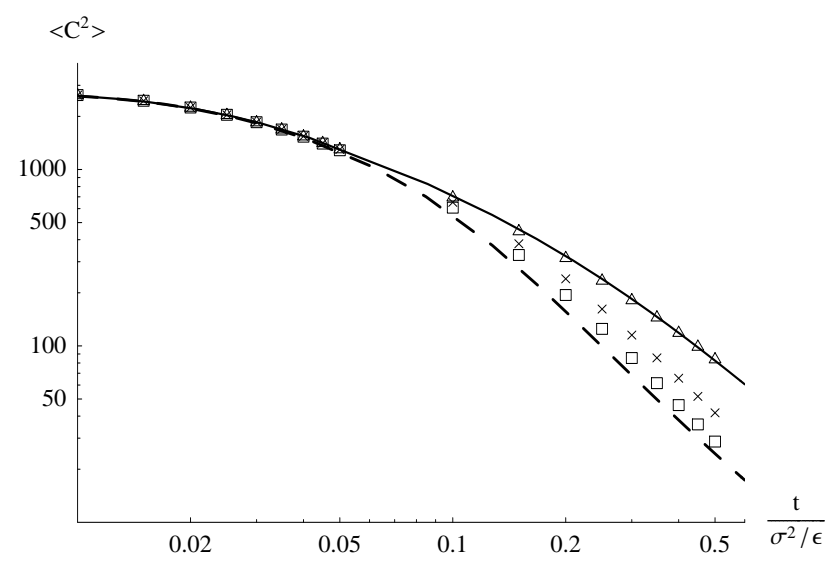

Fig. 5. Comparison between concentration fluctuations predicted by the 1-D $(\triangle), 2-\mathrm{D}(\times)$ and 3-D $(\square)$ models and theoretical prediction based on the Richardson (solid line) and Gaussian (dashed line) PDFs.

not Gaussian. Thus the 2-D and 3-D models are not able to predict very small particle separations.

For time smaller than $t_{0}$ the PDF can be considered Gaussian. This result agrees with the theoretical predictions by Batchelor (1952) and Richardson (1926).

We found that the separation PDF is Gaussian at small times, for $t \ll t_{0}$ and outside the inertial subrange $\left(t \gg T_{\mathrm{L}}\right)$, but it departs from Gaussianity in the intermediate subrange $\left(t_{0} \ll t \ll T_{\mathrm{L}}\right)$. In this range the PDF agrees, at least for small separation, with those proposed by Thomson (Eq. 13) (Thomson, 1990; Monin and Yaglom, 1975). In the case of the 1-D model it agrees with the Richardson PDF (Eq. 12), (Ferrero and Mortarini, 2005).

\section{Concentrations}

We have shown that the PDF of the separation is not the same along all the inertial range, so using the Gaussian PDF for calculating the concentration and the concentration fluctuations can not always be correct. We derived a new formula based on the Richardson PDF and we compared this formula with that proposed by Thomson (1990) based on the Gaussian PDF.

The mean concentrations and the concentration fluctuations predicted by the model are compared with the analytical formulas based on the single particle $\left(P_{1}(y, s \mid x, t)\right)$ and two particles separation $\left(P_{2}\left(y_{1}, y_{2}, s, s \mid x_{1}, x_{2}, t, t\right)\right)$ PDFs, respectively.

In order to calculate the mean concentrations and concentration fluctuations we adopted the Sawford (1993) approximation, obtaining the following expressions

$\langle C(x, t)\rangle=\int \frac{1}{\sqrt{2 \pi} \sigma_{1}(s \mid t)} e^{-\frac{(x-y)^{2}}{2 \sigma_{1}^{2}(s \mid t)}} S(y) d y$ 


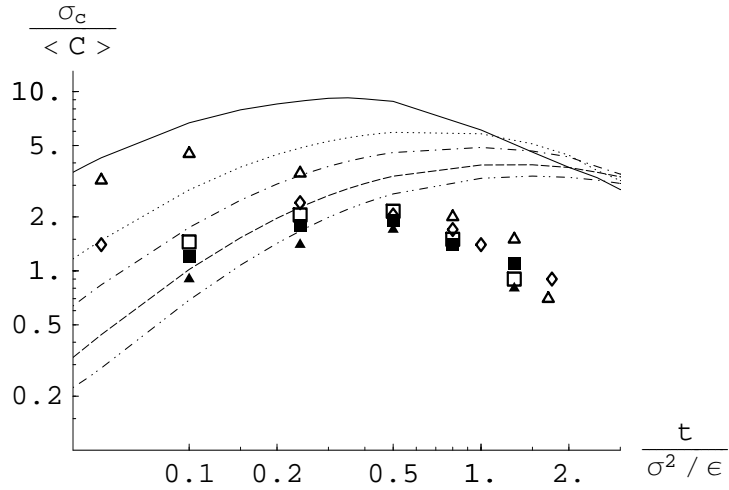

(a)

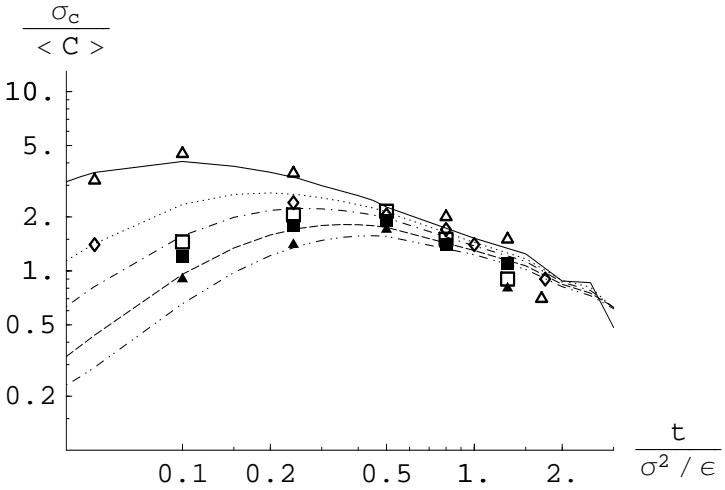

(b)

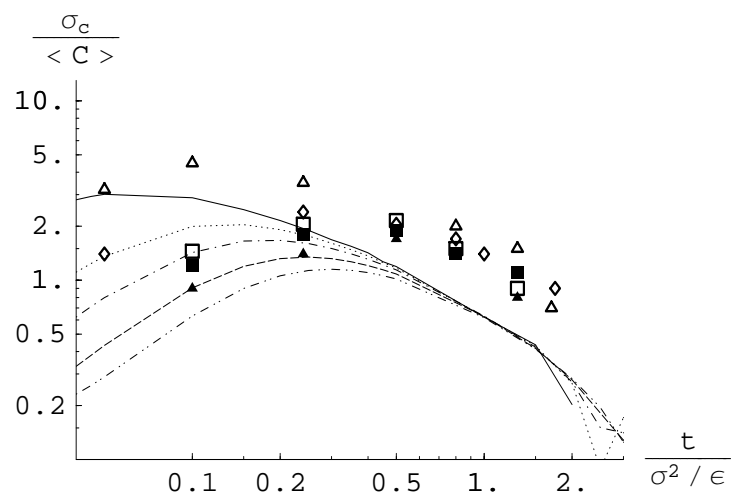

(c)

Fig. 6. Comparison between model simulations (lines) and experimental data (symbols) for different source sizes; (a): 1-D model, (b): 2-D model, (c): 3-D model.

$\left\langle C^{2}(x, t)\right\rangle=\int P(\Delta, s \mid t) \frac{1}{\sqrt{2 \pi} \sigma_{\Sigma}(s \mid t)} e^{-\frac{(x \sqrt{2}-\Sigma)^{2}}{2 \sigma_{\Sigma}^{2}(s \mid t)}}$

$S\left(y_{1}\right) S\left(y_{2}\right) d y_{1} d y_{2}$

where $S(y)$ is the amount of tracer released per unit volume, $P(\Delta, s \mid t)$ the separation PDF, $\sigma_{1}$ is the single particle position standard deviation and $\sigma_{\Sigma}$ is the barycentre standard deviation.

For the sake of simplicity, we consider a discrete Gaussian area source (where $\sigma_{0}$ represents the source size):

$S(y)=\frac{1}{\sqrt{2 \pi} \sigma_{0}} e^{-\frac{y^{2}}{2 \sigma_{0}^{2}}}$

which gives the following expressions for the mean concentration:

$$
\langle C(x, t)\rangle=\frac{1}{\sqrt{2 \pi\left(\sigma_{1}^{2}(s \mid t)+\sigma_{0}^{2}\right)}} e^{-\frac{x^{2}}{2\left(\sigma_{1}^{2}(s \mid t)+\sigma_{0}^{2}\right)}}
$$

Concerning the mean concentration fluctuations we derived the following expression:

$$
\left\langle C^{2}(x, t)\right\rangle=A\left(\eta, \sigma_{0}, t\right) \frac{3 e^{-\frac{x^{2}}{\sigma_{\Sigma}^{2}(t)+\sigma_{0}^{2}}}}{2^{6} 2^{5 / 6} \pi^{2} \sqrt{t^{7} \eta\left(\sigma_{\Sigma}^{2}(t)+\sigma_{0}^{2}\right)}}
$$

Mean concentrations predicted by the models for an area source were calculated and compared with the predictions based on the Gaussian PDF (Eq. 17) see Fig. 4. The results obtained with all the three models agree with the theoretical predictions. It can also be observed that the mean concentrations corresponding to the three models follow the same behavior and, after about 0.1 integral time scale $\left(\sigma^{2} / \epsilon\right)$, the predictions, for sources with different size, collapse on the same curve. This demonstrates that the characteristics of the source influence the dispersion only at the shortest times.

Concerning the concentration fluctuation, the 1-D, 2-D and 3-D models give different results, according to the different shapes of the separation PDF. In Fig. 5 the model results are compared with the theoretical predictions obtained from Eq. (18) and those from the analogous formula based on the Gaussian PDF (Thomson, 1990). The curve corresponding 
to Eq. (18) slightly differs in the cases of 1-D, 2-D and 3-D models. As a matter of fact the value of $\eta$ depends on which of the models is considered. As long as the differences are not significative, in Fig. 5 we only plot the curve corresponding to the 1-D model.

In the 1-D case the calculated values agree with the theoretical prediction based on the Richardson PDF, while the 2-D and 3-D models show small differences from the Gaussian behavior only at shorter times (more pronounced for the 2-D model).

A comparison of the model results with measured data is shown in Fig. 6. The experiment was conducted in a wind tunnel by Fackrell and Robins (1982). A continuous line source is compared with an instantaneous area source, so in the 1-D case two coupled equations were considered.

The results show that concentration fluctuations predicted by the three models are different and depend on the separation PDF. It can be observed that the highest values are predicted by the 1-D model while the 3-D model gives the lowest concentration fluctuations.

It should be stressed that the results depend on the choice of the constant $C_{0}$, in the simulation here presented we set $C_{0}=4$. However, a higher value of $\left\langle C^{2}>\right.$ can be obtained by decreasing this value.

\section{Conclusions}

In this paper we show the results obtained by using a Lagrangian Stochastic Model for the particle pairs dispersion, developed following Thomson (1990). The comparison of the separation and barycentre mean squares with the theoretical behaviour, agrees with the Richardson $\mathrm{t}^{3}$-law and furthermore seems to confirm the presence of the intermediate subrange for $t_{0} \ll t \ll T_{\mathrm{L}}$, where $t_{0}$ is determined by the particles initial separation as suggested by Batchelor (1952) and Borgas and Sawford (1991). Even with the 3-D model the barycentre mean square trend does not agree with the theoretical prediction, Eq. (10), as shown in the 1-D case by Ferrero and Mortarini (2005), confirming the different role played by the non linear terms in the two Langevin equations.

Regarding the PDF of the separation we found that for $t \ll t_{0}$ and $t \gg T_{\mathrm{L}}$ it is Gaussian, confirming the (Batchelor, $1952)$ results, while in the intermediate subrange $\left(t_{0} \ll t \ll T_{\mathrm{L}}\right)$ it departs from the Gaussian distribution. The 1-D model gives a Richardson PDF for the separation in the intermediate range (Ferrero and Mortarini, 2005), while in the case of the 2-D and 3-D models the separation PDF shows a less sharp maximum reproducing the asymptotic behavior for small separation suggested by Thomson (1990).

The different separation PDFs provide different concentration fluctuations and the Gaussian approximation seems to be not applicable particularly at the short times.

Edited by: L. M. Frohn

\section{References}

Batchelor, G. K.: Diffusion in a field of homogeneous turbulence. II. The relative motion of particles, Proc. Cam. Phil. Soc., 48, 345-362, 1952.

Borgas, M. S. and Sawford, B. L.: The small-scale structure of acceleration correlation and its role in the statistical theory of turbulence dispersion, J. Fluid Mech., 228, 295-320, 1991.

Borgas, M. S. and Sawford, B. L.: A Family of Stochastic Models For Particle Dispersion in Isotropic Homogeneous Stationary Turbulence, J. Fluid Mech., 279, 69-99, 1994.

Crone, G. C., Dinar, N., van Dop, H., and Verwer, G. H. L.: A Lagrangian approach for modelling turbulent transport and chemistry, Atmos. Environ., 33, 4919-4934, 1999.

Du, S., Wilson, J. D., and Yee, E.: Probability density functions for velocity in the convective boundary layer and implied trajectory models, Atmos. Environ., 28, 1211-1217, 1994.

Durbin, P.: A stochastic model of two particle dispersion and concentration fluctuation in homogeneous turbulence, J. Fluid Mech., 100, 279-302, 1980.

Fackrell, J. E. and Robins, A. G.: Concentration fluctuations and fluxes in plumes from point sources in a turbulent boundary, J. Fluid Mech., 117, 1-26, 1982.

Ferrero, E. and Mortarini, L.: Concentration fluctuations and relative dispersion PDF, Atmos. Environ., 39, 11, 2135-2143, 2005.

Ferrero, E., Castelli, S. T., and Anfossi, D.: Turbulence fields for atmospheric dispersion models in horizontally nonhomogeneous conditions, Atmos. Environ., 37, 2305-2315, 2003.

Hurley, P. and Physick, W.: A skewed homogeneous Lagrangian particle model for convective conditions, Atmos. Environ., 25A, 1313-1325, 1991.

Kaplan, H. and Dinar, N.: Diffusion of an instantaneous cluster of particles in homogeneous turbulence field, J. Fluid Mech., 203, 273-287, 1989.

Lee, J. T. and Stone, G. L.: The use of Eulerian initial condition in a Lagrangian model of turbulent diffusion, Atmos. Environ., 17, 2477-2481, 1983.

Luhar, A. K. and Britter, R. E.: A random walk model for dispersion in inhomogeneous turbulence in convective boundary layer, Atmos. Environ., 23, 1911-1924, 1989.

Monin, A. and Yaglom, A.: Statistical fluid mechanics, vol. 2, MIT Press, Cambridge, Mass, 1975.

Richardson, L. F.: Atmospheric diffusion shown on a distanceneighbour graph, Proc. Roy. Soc., A 110, 709-737, 1926.

Sawford, B. L.: The basis for, and some limitations of, the Langevin Equation in Atmospheric Relative dispersion modelling, Atmos. Environ, 18, 2405-2411, 1984.

Sawford, B. L.: Recent development in the Lagrangian stochastic theory of turbulent dispersion, Bound. Layer Meteor., 63, 197215, 1993.

Taylor, G. I.: Diffusion by continuous movements, Proc. London Math. Soc., 20, 196-211, 1921.

Thomson, D. J.: Criteria for the selection of stochastic models of particle trajectories in turbulent flows, J. Fluid Mech., 180, 529556, 1987.

Thomson, D. J.: A stochastic model for the motion of particle pairs in isotropic high Reynolds number, and its application to the problem of concentration variance, J. Fluid Mech., 210, 113153, 1990. 
Tinarelli, G., Anfossi, D., Brusasca, G., Ferrero, E., Giostra, U., Morselli, M. G., Moussafir, J., Tampieri, F., and Trombetti, F.: Lagrangian particle simulation of tracer dispersion in the lee of a schematic twodimensional hill, J. App. Met., 33, 744-756, 1994. van Dop, H.: The evaluation of a lagrangian model for turbulent transport and chemistry, Phys. of Fluids, 13, 1331-1342, 2001. 